\title{
Lie-algebraic stability conditions for nonlinear switched systems and differential inclusions
}

\author{
Michael Margaliot* \\ Dept. of Electrical Engineering - Systems \\ Tel Aviv University \\ Tel Aviv 69978, Israel
}

\author{
Daniel Liberzon ${ }^{\dagger}$ \\ Coordinated Science Laboratory \\ Univ. of Illinois at Urbana-Champaign \\ Urbana, IL 61821, U.S.A.
}

\begin{abstract}
We present a stability criterion for switched nonlinear systems which involves Lie brackets of the individual vector fields but does not require that these vector fields commute. A special case of the main result says that a switched system generated by a pair of globally asymptotically stable nonlinear vector fields whose third-order Lie brackets vanish is globally uniformly asymptotically stable under arbitrary switching. This generalizes a known fact for switched linear systems and provides a partial solution to the open problem posed in [11]. To prove the result, we consider an optimal control problem which consists in finding the "most unstable" trajectory for an associated control system, and show that there exists an optimal solution which is bang-bang with a bound on the total number of switches. This property is obtained as a special case of a reachability result by bang-bang controls which is of independent interest. By construction, our criterion also automatically applies to the corresponding relaxed differential inclusion.
\end{abstract}

Keywords: Switched nonlinear system, global asymptotic stability, Lie bracket, optimal control, maximum principle, differential inclusion.

\section{Introduction}

A finite family of smooth vector fields $\boldsymbol{f}_{0}, \boldsymbol{f}_{1}, \ldots, \boldsymbol{f}_{m}$ on $\mathbb{R}^{n}$ gives rise to the switched system

$$
\dot{\boldsymbol{x}}=\boldsymbol{f}_{\sigma}(\boldsymbol{x}), \quad \boldsymbol{x} \in \mathbb{R}^{n} .
$$

Here $\sigma:[0, \infty) \rightarrow\{0,1, \ldots, m\}$ is a piecewise constant function of time, called a switching signal. A frequently discussed example, which we will occasionally use for illustration, is when the given vector fields are linear: $\boldsymbol{f}_{i}(\boldsymbol{x})=A_{i} \boldsymbol{x}$, where $A_{i}$ is an $n \times n$ matrix, $i=0,1, \ldots, m$. This yields the switched linear system

$$
\dot{\boldsymbol{x}}=A_{\sigma} \boldsymbol{x}, \quad \boldsymbol{x} \in \mathbb{R}^{n} .
$$

Switched systems have numerous applications and represent a subject of extensive ongoing research (see, e.g., [10] and the many references therein).

By a solution of (1) we understand an absolutely continuous $\mathbb{R}^{n}$-valued function $\boldsymbol{x}(\cdot)$ which satisfies the differential equation for almost all $t$ in its domain. In the context of switched systems, one usually assumes

\footnotetext{
${ }^{*}$ Supported by the ISF under grant number 199/03. Email: michaelm@eng.tau.ac.il

${ }^{\dagger}$ Corresponding author. Supported by NSF ECS-0134115 CAR, NSF ECS-0114725, and DARPA/AFOSR MURI F4962002-1-0325 grants. Fax: (217) 244-2352. Email: liberzon@uiuc.edu
} 
for simplicity that the switching signal $\sigma$ has a finite number of discontinuities, or switches, on every bounded time interval. Our results will in fact automatically apply to measurable switching signals and also to solutions of the relaxed differential inclusion associated with (1). This is the differential inclusion

$$
\dot{\boldsymbol{x}} \in \operatorname{co}\left\{\boldsymbol{f}_{0}(\boldsymbol{x}), \boldsymbol{f}_{1}(\boldsymbol{x}), \ldots, \boldsymbol{f}_{m}(\boldsymbol{x})\right\}
$$

where co denotes the convex hull. Its solutions are absolutely continuous functions $\boldsymbol{x}(\cdot)$ satisfying (3) for almost all $t$, and this includes all solutions of the switched system (1) with piecewise constant $\sigma$ (see, e.g., [4] for more information on differential inclusions).

When we refer to stability of the switched system (1), the precise property of interest will be global asymptotic stability which is uniform over the set of all switching signals, formally defined as follows. The switched system (1) is called globally uniformly asymptotically stable (GUAS) if there exists a class $\mathcal{K} \mathcal{L}$ function ${ }^{1} \beta$ such that for every switching signal $\sigma$ and every initial condition $\boldsymbol{x}(0)$ the corresponding solution of (1) satisfies

$$
|\boldsymbol{x}(t)| \leq \beta(|\boldsymbol{x}(0)|, t) \quad \forall t \geq 0
$$

(here and below, $|\cdot|$ denotes the standard Euclidean norm on $\mathbb{R}^{n}$ ). An equivalent definition can be given in the $\epsilon-\delta$ style (cf. [13]). If the function $\beta$ in (4) takes the form $\beta(r, t)=a r e^{-b t}$ for some $a, b>0$, then the switched system (1) is called globally uniformly exponentially stable (GUES). The above definitions reduce to the usual notions of global asymptotic and exponential stability in the case of a single system. If (4) holds along all solutions of the differential inclusion (3), then this differential inclusion is called globally asymptotically stable. This property implies GUAS of the switched system (1) because solutions of (1) are contained in those of (3).

It is well known and easy to demonstrate that global asymptotic stability of the individual subsystems $\dot{\boldsymbol{x}}=\boldsymbol{f}_{i}(\boldsymbol{x}), i=0,1, \ldots, m$ is necessary but not sufficient for global asymptotic stability of the switched system (1) for every possible switching signal, let alone GUAS. In this paper, we are concerned with the problem of identifying conditions on the individual subsystems - besides the obviously necessary requirement of their global asymptotic stability - which guarantee GUAS of (1). This problem has received considerable attention in the literature; see [10, Chapter 2] for some available results.

We now review two directions of attack on the GUAS problem. The difficulty in analyzing the stability of (1) is that the switched system admits an infinite number of solutions for each initial condition. A natural idea is to try to characterize the "worst-case" (that is, the "most unstable") switching law, and then analyze the behavior of the unique trajectory produced by this law. Pyatnitskiy and Rapoport $[19,20]$ developed a variational approach to describe the "worst-case" switching law, and the corresponding trajectory, for the switched linear system (2). Applying the Maximum Principle, they derived an implicit characterization of this switching law in terms of a two-point boundary value problem. More recently, the same variational problem was addressed using a dynamic programming approach [15]. For the particular case of secondorder switched linear systems, this approach yields an easily verifiable necessary and sufficient condition for GUAS [16] (see also [8] as well as the related work [5]).

Another particularly promising research avenue is to explore the role of commutation relations among the subsystems being switched. We now briefly recall available results, starting with the case of the switched linear system (2). The commutator, or Lie bracket, is defined as $\left[A_{i}, A_{j}\right]:=A_{i} A_{j}-A_{j} A_{i}$. First, suppose that the matrices commute: $\left[A_{i}, A_{j}\right]=0$ for all $i, j$. Then it is well known that the system $(2)$ is GUES provided that $A_{1}, \ldots, A_{m}$ are all Hurwitz. (This is not hard to show by a direct analysis of the

\footnotetext{
${ }^{1}$ Recall that a function $\alpha:[0, \infty) \rightarrow[0, \infty)$ is said to be of class $\mathcal{K}$ if it is continuous, strictly increasing, and $\alpha(0)=0$. A function $\beta:[0, \infty) \times[0, \infty) \rightarrow[0, \infty)$ is said to be of class $\mathcal{K} \mathcal{L}$ if $\beta(\cdot, t)$ is of class $\mathcal{K}$ for each fixed $t \geq 0$ and $\beta(s, t)$ decreases to 0 as $t \rightarrow \infty$ for each fixed $s \geq 0$.
} 
transition matrix, or by constructing a quadratic common Lyapunov function via the iterative procedure proposed in [17].) It was shown in [7] that for $m=1$ the above statement remains true if the Lie bracket condition is relaxed to $\left[A_{0},\left[A_{0}, A_{1}\right]\right]=\left[A_{1},\left[A_{0}, A_{1}\right]\right]=0$. This result was extended in [12] to all nilpotent and solvable matrix Lie algebras for arbitrary $m$ by using simultaneous triangularization (Lie's Theorem), and later in [1] to a more general class of matrix Lie algebras; the latter paper also established that no further generalization based on the Lie bracket relations alone is possible.

Let us now return to the nonlinear setting described by the switched system (1), which is much less thoroughly understood. Linearizing the individual subsystems and applying the previous results together with Lyapunov's indirect method, it is not difficult to obtain conditions for local asymptotic stability of $(1)$; this was done in $[12,1]$. To tackle the global stability question, one can try to inspect commutation relations between the original nonlinear vector fields $\boldsymbol{f}_{0}, \boldsymbol{f}_{1}, \ldots, \boldsymbol{f}_{m}$. The Lie bracket is now defined as

$$
\left[\boldsymbol{f}_{i}, \boldsymbol{f}_{j}\right](\boldsymbol{x}):=\frac{\partial \boldsymbol{f}_{j}(\boldsymbol{x})}{\partial \boldsymbol{x}} \boldsymbol{f}_{i}(\boldsymbol{x})-\frac{\partial \boldsymbol{f}_{i}(\boldsymbol{x})}{\partial \boldsymbol{x}} \boldsymbol{f}_{j}(\boldsymbol{x}) .
$$

It turns out that if the two vector fields commute, i.e., if $\left[\boldsymbol{f}_{i}, \boldsymbol{f}_{j}\right](\boldsymbol{x}) \equiv \mathbf{0}$ for all $i, j$, then global asymptotic stability of the individual subsystems still implies GUAS of the switched system (1). Similarly to the linear case, this can be proved either by direct analysis of the flow (see [14]) or by an iterative construction of a common Lyapunov function in the spirit of [17] (see [21] and [27]). All attempts to formulate global asymptotic stability criteria valid beyond the commuting nonlinear case have so far been unsuccessful, as the methods employed to obtain the corresponding results for switched linear systems do not seem to apply. These issues are explained in [11], where this is proposed as an open problem for which an altogether different approach seems to be required.

Such a different approach is proposed in this paper. It is based on merging the two directions outlined above; namely, it combines the variational approach with a Lie-algebraic analysis of the "worst-case" switching law. It is a well-known fact that Lie brackets play an essential role in the Maximum Principle of optimal control (see, e.g., [24]). In the present context, it turns out that the "worst-case" switching law for the switched nonlinear system (1) is governed by the signs of suitable functions of time whose derivatives are determined by Lie brackets of $\boldsymbol{f}_{0}, \boldsymbol{f}_{1}, \ldots, \boldsymbol{f}_{m}$. We impose conditions on these Lie brackets which guarantee that each of these functions is a polynomial. This leads to a bound on the total number of switches for the "worst-case" switching law, and GUAS of the switched system can be deduced from global asymptotic stability of the individual subsystems.

In fact, we show that our Lie-algebraic conditions imply the following general reachability result. Suppose that there exist $\boldsymbol{p}, \boldsymbol{q} \in \mathbb{R}^{n}$, a time $t \geq 0$, and a trajectory of (3) satisfying $\boldsymbol{x}(0)=\boldsymbol{p}$ and $\boldsymbol{x}(t)=\boldsymbol{q}$. Then there exists a trajectory $\boldsymbol{x}(\cdot)$ of $(1)$, with no more than $N$ switchings, which also satisfies $\boldsymbol{x}(0)=\boldsymbol{p}$ and $\boldsymbol{x}(t)=\boldsymbol{q}$. Furthermore, the bound $N$ is uniform over all trajectories and all $t$. This theoretical result has the important practical implication that point-to-point control problems reduce to determining a set of $2 N+1$ parameters: $N$ switching times and $N+1$ control values (this relies on a well-known equivalence between differential inclusions and control systems, discussed in the next section). There is a rich literature on conditions guaranteeing that optimal controls are, in some sense, regular (e.g., finite combinations of bang-bang and singular arcs); cf. [22, 24] and the references therein. However, these results are typically local, in the sense that the value of the bound $N$ depends on the length of the time interval.

We now formally state our main result. We denote by $(\operatorname{ad} \boldsymbol{f})^{r}, r=0,1, \ldots$ the operators defined by $(\operatorname{ad} \boldsymbol{f})^{0}(\boldsymbol{g}):=\boldsymbol{g}$ and $(\operatorname{ad} \boldsymbol{f})^{r}(\boldsymbol{g}):=\left[\boldsymbol{f},(\operatorname{ad} \boldsymbol{f})^{r-1}(\boldsymbol{g})\right]$ for $r \geq 1$, where $\boldsymbol{f}$ and $\boldsymbol{g}$ are smooth vector fields on $\mathbb{R}^{n}$. The analyticity assumption in the theorem can be relaxed, as explained in Remark 7 in Section 4 . See also Remark 2 in Section 2 for a different version of this result. 
Theorem 1 Suppose that $\boldsymbol{f}_{0}, \boldsymbol{f}_{1}, \ldots, \boldsymbol{f}_{m}$ are analytic vector fields on $\mathbb{R}^{n}$ such that the systems $\dot{\boldsymbol{x}}=\boldsymbol{f}_{i}(\boldsymbol{x})$, $i=0,1, \ldots, m$ are globally asymptotically stable and backward complete. ${ }^{2}$ Suppose that there exists a positive integer $r$ such that for every index $l \in\{0,1, \ldots, m\}$ we have

$$
\left(\operatorname{ad} \boldsymbol{f}_{l}\right)^{r}\left(\boldsymbol{f}_{i}\right)(\boldsymbol{x})=\mathbf{0} \quad \forall \boldsymbol{x} \in \mathbb{R}^{n}, \forall i \neq l
$$

and

$$
\left[\boldsymbol{f}_{k}-\boldsymbol{f}_{l},\left(\operatorname{ad} \boldsymbol{f}_{l}\right)^{s}\left(\boldsymbol{f}_{i}-\boldsymbol{f}_{l}\right)\right](\boldsymbol{x})=\mathbf{0} \quad \forall \boldsymbol{x} \in \mathbb{R}^{n}, \forall i, k \neq l, s=0, \ldots, r-1 .
$$

Then the differential inclusion (3) is globally asymptotically stable, and in particular the switched system (1) is GUAS.

For example, it is easy to check that for $r=1$, the conditions (5) and (6) are equivalent to $\left[\boldsymbol{f}_{i}, \boldsymbol{f}_{j}\right](\boldsymbol{x}) \equiv \mathbf{0}$ for all $i, j$, and we recover the known result for commuting vector fields [14]. The first interesting situation is when $r=2$. It is not difficult to verify that the conditions (5) and (6) then reduce to

$$
\left[\boldsymbol{f}_{k},\left[\boldsymbol{f}_{j}, \boldsymbol{f}_{i}\right]\right](\boldsymbol{x})=\mathbf{0} \quad \forall \boldsymbol{x} \in \mathbb{R}^{n}, \forall i, j, k \in\{0,1, \ldots, m\}
$$

and

$$
\left[\boldsymbol{f}_{k}-\boldsymbol{f}_{j}, \boldsymbol{f}_{i}-\boldsymbol{f}_{j}\right](\boldsymbol{x})=\mathbf{0} \quad \forall \boldsymbol{x} \in \mathbb{R}^{n}, \forall i, j, k \in\{0,1, \ldots, m\} .
$$

Note that in the particular case when $m=1$ (i.e., the case of two subsystems) the last condition is trivially satisfied, and we arrive at the following nonlinear extension of the result proved in [7]. ${ }^{3}$

Corollary 1 Suppose that $\boldsymbol{f}_{0}, \boldsymbol{f}_{1}$ are analytic vector fields on $\mathbb{R}^{n}$ such that the systems $\dot{\boldsymbol{x}}=\boldsymbol{f}_{i}(\boldsymbol{x}), i=0,1$ are globally asymptotically stable and backward complete, and suppose that

$$
\left[\boldsymbol{f}_{0},\left[\boldsymbol{f}_{0}, \boldsymbol{f}_{1}\right]\right](\boldsymbol{x})=\left[\boldsymbol{f}_{1},\left[\boldsymbol{f}_{0}, \boldsymbol{f}_{1}\right]\right](\boldsymbol{x})=\mathbf{0} \quad \forall \boldsymbol{x} \in \mathbb{R}^{n}
$$

Then the differential inclusion (3) is globally asymptotically stable, and in particular the switched system (1) is GUAS.

The remainder of the paper is devoted to proving Theorem 1. In the next section we formulate the "worst-case" optimal control problem associated with (1) and note some of its basic properties. In Section 3 we obtain a key bound on the total number of switches for bang-bang controls, which is used in Section 4 to establish the main result. Section 5 contains some concluding remarks.

\section{Optimal control approach}

Our starting point is to replace the differential inclusion (3) by the control system with drift

$$
\dot{\boldsymbol{x}}=\boldsymbol{f}_{0}(\boldsymbol{x})+\sum_{k=1}^{m} \boldsymbol{g}_{k}(\boldsymbol{x}) u_{k}, \quad \boldsymbol{u}=\left(u_{1}, \ldots, u_{m}\right)^{T} \in \mathcal{U}
$$

where

$$
\boldsymbol{g}_{k}(\boldsymbol{x}):=\boldsymbol{f}_{k}(\boldsymbol{x})-\boldsymbol{f}_{0}(\boldsymbol{x}), \quad k=1, \ldots, m
$$

\footnotetext{
${ }^{2}$ Backward completeness means that all solutions are well defined for arbitrary negative times.

${ }^{3}$ Leonid Gurvits has informed us that he recently also obtained a generalization of his original result to nonlinear systems, using a technique different from ours.
} 
and $\mathcal{U}$ is the standard simplex

$$
\mathcal{U}:=\left\{\boldsymbol{u} \in \mathbb{R}^{m}: u_{k} \geq 0, \sum_{k=1}^{m} u_{k} \leq 1\right\} .
$$

Throughout the rest of the paper, admissible control inputs for (8) will be measurable functions of time taking values in $\mathcal{U}$ (i.e., the control $\boldsymbol{u}(t)=\left(u_{1}(t), \ldots, u_{m}(t)\right)^{T}$ belongs to $\mathcal{U}$ for all $t$ ). The equivalence between the control system (8) and the differential inclusion (3) is revealed by the result stated next, which is well known as Filippov's Selection Lemma (see, e.g., [4, Section 14, Corollary 1] or [26, Theorem 2.3.13]).

Lemma 1 The set of solutions of the control system (8) coincides with the set of solutions of the differential inclusion (3).

Note that trajectories of the original switched system (1) correspond to piecewise constant controls taking values in the set of $m+1$ vertices of $\mathcal{U}$. In particular, $\dot{\boldsymbol{x}}=\boldsymbol{f}_{0}(\boldsymbol{x})$ results by setting $\boldsymbol{u} \equiv \mathbf{0}$ in (8), while each $\dot{\boldsymbol{x}}=\boldsymbol{f}_{i}(\boldsymbol{x}), i=1, \ldots, m$ results by setting $u_{i} \equiv 1$ and $u_{j} \equiv 0, j \neq i$. (The choice of $\boldsymbol{f}_{0}$ as the drift vector field simplifies the notation, but is otherwise completely arbitrary.) We also remark that the switched linear system (2) is associated in this way with the bilinear control system $\dot{\boldsymbol{x}}=A_{0} \boldsymbol{x}+\sum_{k=1}^{m}\left(A_{k} \boldsymbol{x}-A_{0} \boldsymbol{x}\right) u_{k}$.

Fix an arbitrary point $\boldsymbol{p} \in \mathbb{R}^{n}$. Since the right-hand side of (8) is bounded on every bounded ball in $\mathbb{R}^{n}$, we can find a time $T$ (which depends on $|\boldsymbol{p}|$ ) such that all solutions of (8) starting at $\boldsymbol{p}$ are bounded by, say, $2|\boldsymbol{p}|$ for all $t \in[0, T]$. In particular, these solutions are defined at least up to time $T$. Thus there exists the largest time $T_{\max }(|\boldsymbol{p}|) \leq \infty$ such that all solutions of (8) starting at $\boldsymbol{p}$ are defined for $t \in\left[0, T_{\max }\right)$.

Remark 1 Note that since the systems $\dot{\boldsymbol{x}}=\boldsymbol{f}_{i}(\boldsymbol{x}), i=0,1, \ldots, m$ are assumed to be globally asymptotically stable, solutions of (8) corresponding to piecewise constant controls taking values in the set of vertices of $\mathcal{U}$ automatically exist for all $t \geq 0$ (these are solutions of the original switched system). However, this is not a priori true for arbitrary measurable controls taking values in $\mathcal{U}$, unless $\boldsymbol{f}_{0}, \boldsymbol{f}_{1}, \ldots, \boldsymbol{f}_{m}$ satisfy suitable growth conditions (such as $\left|\boldsymbol{f}_{i}(\boldsymbol{x})\right| \leq r(|\boldsymbol{x}|+c), r, c>0$; see, e.g., [26, p. 92]). Although we will show later that under the hypotheses of Theorem 1 solutions of (8) indeed exist globally, for the moment we need to work with sufficiently small time intervals. See also Remark 6 in Section 4.

Let $\boldsymbol{x}(\cdot ; \boldsymbol{p}, \boldsymbol{u})$ denote the solution of the system (8) with initial condition $\boldsymbol{x}(0)=\boldsymbol{p}$ corresponding to an admissible control $\boldsymbol{u}$. Picking a positive final time $t_{f}<T_{\max }(|\boldsymbol{p}|)$, we define

$$
J\left(t_{f}, \boldsymbol{p}, \boldsymbol{u}\right):=\left|\boldsymbol{x}\left(t_{f} ; \boldsymbol{p}, \boldsymbol{u}\right)\right|^{2} .
$$

We pose the following optimal control problem in terms of the cost functional $J$.

Problem 1 Find a control $\boldsymbol{u}$ that maximizes $J$ along the solutions of (8).

We note that this problem is well posed, i.e., an optimal control does exist.

Lemma 2 Problem 1 admits a solution for every pair $\left(\boldsymbol{p}, t_{f}\right)$ such that $t_{f}<T_{\max }(|\boldsymbol{p}|)$.

Proof. By Lemma 1, solutions of the control system (8) coincide with solutions of the differential inclusion (3). We can thus apply [6, $\S 7$, Theorem 3] and conclude that the set of these solutions with $\boldsymbol{x}(0)=\boldsymbol{p}$ for $0 \leq t \leq t_{f}$ is compact with respect to the topology of uniform convergence. The functional $J$ represents a continuous functional on this set. Therefore, its maximum is well defined. 
The intuitive interpretation of Problem 1 is clear: find a control that "pushes" the state as far away from the origin as possible (from a given initial condition in a given amount of time). If we can show that the resulting closed-loop system is stable, then the same property should hold for all other controls, and stability of the original switched system - as well as the differential inclusion - will be established. This program will be formally carried out in what follows.

Consider an optimal control $\tilde{\boldsymbol{u}}=\left(\tilde{u}_{1}, \ldots, \tilde{u}_{m}\right)^{T}$ and the corresponding optimal trajectory $\tilde{\boldsymbol{x}}$. We will study properties of $\tilde{\boldsymbol{u}}$ using the Maximum Principle (see, e.g, [2, 9] for background on the Maximum Principle of optimal control, in a geometric framework suitable for our subsequent developments). The Hamiltonian for Problem 1 is

$$
H(\boldsymbol{x}, \boldsymbol{u}, \boldsymbol{\lambda}):=\boldsymbol{\lambda}^{T} \boldsymbol{f}_{0}(\boldsymbol{x})+\sum_{k=1}^{m} \boldsymbol{\lambda}^{T} \boldsymbol{g}_{k}(\boldsymbol{x}) u_{k}
$$

where $\boldsymbol{\lambda}:\left[0, t_{f}\right] \rightarrow \mathbb{R}^{n}$ is a costate satisfying the adjoint equation associated with (8):

$$
\dot{\boldsymbol{\lambda}}=-\frac{\partial H}{\partial \boldsymbol{x}}=-\left(\frac{\partial \boldsymbol{f}_{0}}{\partial \boldsymbol{x}}\right)^{T} \boldsymbol{\lambda}-\sum_{k=1}^{m}\left(\frac{\partial \boldsymbol{g}_{k}}{\partial \boldsymbol{x}}\right)^{T} \boldsymbol{\lambda} u_{k} .
$$

More precisely, $\boldsymbol{\lambda}$ is identified with the linear functional on $\mathbb{R}^{n}$ defined via the inner product $\langle\boldsymbol{\lambda}(t), \boldsymbol{\xi}\rangle=$ $\boldsymbol{\lambda}^{T}(t) \boldsymbol{\xi}$. (In the more general formulation of the Maximum Principle for manifolds, $\boldsymbol{\lambda}(t)$ is a linear functional on the tangent space at $\boldsymbol{x}(t)$; its evolution is described by the adjoint equation, which can be defined intrinsically and is given by (11) in local coordinates.) The necessary condition for optimality provided by the Maximum Principle states that $\tilde{\boldsymbol{u}}(t), t \in\left[0, t_{f}\right]$ must pointwise maximize $H(\tilde{\boldsymbol{x}}(t), \cdot \tilde{\boldsymbol{\lambda}}(t))$ for the costate $\tilde{\boldsymbol{\lambda}}$ associated with the optimal trajectory satisfying the boundary condition $\tilde{\boldsymbol{\lambda}}\left(t_{f}\right)=2 \tilde{\boldsymbol{x}}\left(t_{f}\right)$. For $i=1, \ldots, m$, let us define the functions $\psi_{i}: \mathbb{R}^{n} \times \mathbb{R}^{n} \rightarrow \mathbb{R}$ by

$$
\psi_{i}(\boldsymbol{x}, \boldsymbol{\lambda}):=\boldsymbol{\lambda}^{T} \boldsymbol{g}_{i}(\boldsymbol{x}) .
$$

Then the optimal control must satisfy the following conditions almost everywhere: for each $i$,

$$
\begin{array}{llll}
\tilde{u}_{i}(t)=0 & \text { if } \quad \psi_{i}(\tilde{\boldsymbol{x}}(t), \tilde{\boldsymbol{\lambda}}(t))<0 & \text { or } \quad \psi_{i}(\tilde{\boldsymbol{x}}(t), \tilde{\boldsymbol{\lambda}}(t))<\psi_{j}(\tilde{\boldsymbol{x}}(t), \tilde{\boldsymbol{\lambda}}(t)) \text { for some } j \neq i \\
\tilde{u}_{i}(t)=1 & \text { if } \quad \psi_{i}(\tilde{\boldsymbol{x}}(t), \tilde{\boldsymbol{\lambda}}(t))>0 \quad \text { and } \quad \psi_{i}(\tilde{\boldsymbol{x}}(t), \tilde{\boldsymbol{\lambda}}(t))>\psi_{j}(\tilde{\boldsymbol{x}}(t), \tilde{\boldsymbol{\lambda}}(t)) \text { for all } j \neq i
\end{array}
$$

If $\psi_{i}(\tilde{\boldsymbol{x}}(t), \tilde{\boldsymbol{\lambda}}(t))=\max \left\{0, \psi_{j}(\tilde{\boldsymbol{x}}(t), \tilde{\boldsymbol{\lambda}}(t)): j \neq i\right\}$, then the Maximum Principle does not yield enough information to uniquely determine $\tilde{u}_{i}(t)$.

We say that a control $\boldsymbol{u}$ is bang-bang if it takes values in the set of vertices of $\mathcal{U}$ and has a finite number of switches on every bounded time interval. It is clear from (13) that if all functions $\psi_{i}(\tilde{\boldsymbol{x}}(\cdot), \tilde{\boldsymbol{\lambda}}(\cdot))$ and $\psi_{i}(\tilde{\boldsymbol{x}}(\cdot), \tilde{\boldsymbol{\lambda}}(\cdot))-\psi_{j}(\tilde{\boldsymbol{x}}(\cdot), \tilde{\boldsymbol{\lambda}}(\cdot)), 1 \leq i, j \leq m$ have isolated zeros on $\left[0, t_{f}\right]$, then the optimal control is bang-bang (modulo a modification on a set of measure 0 which does not change the trajectory). On the other hand, it may happen that one of these functions identically vanishes on some subinterval of $\left[0, t_{f}\right]$, giving rise to a singular optimal trajectory.

The connection between the bang-bang property and Lie brackets comes from the fact that time derivatives of the functions $\psi_{i}, i=1, \ldots, m$ evaluated along state-costate trajectories are expressed in terms of iterated Lie brackets of $\boldsymbol{f}_{0}, \boldsymbol{g}_{1}, \ldots, \boldsymbol{g}_{m}$. This is revealed in the next lemma, which will be crucial in establishing a bound on the total number of switches for bang-bang optimal controls.

Lemma 3 Assume that the hypotheses of Theorem 1 hold. Let $\boldsymbol{x}(\cdot)$ and $\boldsymbol{\lambda}(\cdot)$ be trajectories of (8) and (11) corresponding to some arbitrary control $\boldsymbol{u}$. Then all functions $\psi_{i}(\boldsymbol{x}(\cdot), \boldsymbol{\lambda}(\cdot)), i=1, \ldots, m$ are polynomial functions of time, with degrees not exceeding $r-1$. 
Proof. Pick an arbitrary index $i \in\{1, \ldots, m\}$. To simplify the notation, denote $\psi_{i}(\boldsymbol{x}(t), \boldsymbol{\lambda}(t))$ by $\varphi_{i}(t)$. We know from (12) that $\varphi_{i}$ is absolutely continuous. Differentiating it and using (8) and (11), we find that the derivative is given (almost everywhere) by

$$
\dot{\varphi}_{i}(t)=\boldsymbol{\lambda}^{T}(t)\left[\boldsymbol{f}_{0}, \boldsymbol{g}_{i}\right](\boldsymbol{x}(t))+\sum_{k=1}^{m} \boldsymbol{\lambda}^{T}(t)\left[\boldsymbol{g}_{k}, \boldsymbol{g}_{i}\right](\boldsymbol{x}(t)) u_{k}(t) .
$$

Recalling the definition (9) and applying (6) with $l=0$ and $s=0$, we see that $\left[\boldsymbol{g}_{k}, \boldsymbol{g}_{i}\right] \equiv \mathbf{0}$ for all $i, k$. Thus (14) simplifies to

$$
\dot{\varphi}_{i}(t)=\lambda^{T}(t)\left[\boldsymbol{f}_{0}, \boldsymbol{g}_{i}\right](\boldsymbol{x}(t)) .
$$

It follows from this expression that $\dot{\varphi}_{i}$ is absolutely continuous, and we can differentiate it again. Proceeding in this fashion, we have

$$
\varphi_{i}^{(s+1)}(t)=\boldsymbol{\lambda}^{T}(t)\left(\operatorname{ad} \boldsymbol{f}_{0}\right)^{s+1}\left(\boldsymbol{g}_{i}\right)(\boldsymbol{x}(t))+\sum_{k=1}^{m} \boldsymbol{\lambda}^{T}(t)\left[\boldsymbol{g}_{k},\left(\operatorname{ad} \boldsymbol{f}_{0}\right)^{s}\left(\boldsymbol{g}_{i}\right)\right](\boldsymbol{x}(t)) u_{k}(t), \quad s=0, \ldots, r-1 .
$$

The summation terms involving the control vanish for each $s$ by virtue of (6) with $l=0$, and for $s=r-1$

the first term on the right-hand side also vanishes because of $(5)$ with $l=0$. This means that $\varphi_{i}^{(r)}(t) \equiv 0$, and the claim follows (in view of absolute continuity of $\varphi_{i}$ ).

Remark 2 With the help of the Jacobi identity, it is possible to arrive at an equivalent reformulation of the conditions of Theorem 1 for $r \geq 3$. Namely, let us rewrite the Lie brackets appearing in the summation terms in (15) for $s \geq 2$ using the Jacobi identity as

$$
\left[\boldsymbol{g}_{k},\left(\operatorname{ad} \boldsymbol{f}_{0}\right)^{s}\left(\boldsymbol{g}_{i}\right)\right]=\left[\boldsymbol{g}_{k},\left[\boldsymbol{f}_{0},\left(\operatorname{ad} \boldsymbol{f}_{0}\right)^{s-1}\left(\boldsymbol{g}_{i}\right)\right]\right]=\left[\boldsymbol{f}_{0},\left[\boldsymbol{g}_{k},\left(\operatorname{ad} \boldsymbol{f}_{0}\right)^{s-1}\left(\boldsymbol{g}_{i}\right)\right]\right]+\left[\left(\operatorname{ad} \boldsymbol{f}_{0}\right)^{s-1}\left(\boldsymbol{g}_{i}\right),\left[\boldsymbol{f}_{0}, \boldsymbol{g}_{k}\right]\right]
$$

Thus we can deduce the equality $\left[\boldsymbol{g}_{k},\left(\operatorname{ad} \boldsymbol{f}_{0}\right)^{s}\left(\boldsymbol{g}_{i}\right)\right] \equiv \mathbf{0}$ from the equality $\left[\boldsymbol{g}_{k},\left(\operatorname{ad} \boldsymbol{f}_{0}\right)^{s-1}\left(\boldsymbol{g}_{i}\right)\right] \equiv \mathbf{0}$ if we impose the condition $\left[\left(\operatorname{ad} \boldsymbol{f}_{0}\right)^{s-1}\left(\boldsymbol{g}_{i}\right),\left[\boldsymbol{f}_{0}, \boldsymbol{g}_{k}\right]\right] \equiv \mathbf{0}$ for $s=2, \ldots, r-1$. In terms of the original vector fields, this yields an alternative version of Theorem 1 , in which the condition (6) is retained for $s=0,1$ but replaced for $s=2, \ldots, r-1$ by

$$
\left[\left(\operatorname{ad} \boldsymbol{f}_{l}\right)^{s-1}\left(\boldsymbol{f}_{i}-\boldsymbol{f}_{l}\right),\left[\boldsymbol{f}_{l}, \boldsymbol{f}_{k}\right]\right](\boldsymbol{x})=\mathbf{0} \quad \forall \boldsymbol{x} \in \mathbb{R}^{n}, \forall i, k \neq l .
$$

Together with (5), this results in a set of conditions equivalent to those of Theorem 1.

The main difficulty that remains is the possibility of singular optimal trajectories. As we show next, this problem can be overcome with the help of a construction due to Sussmann [23].

\section{Bang-bang property}

In this section we first summarize the basic steps of the construction given by Sussmann in [23], adopted to our context, and then show that the present assumptions lead to a more specific result which will play an important role in the proof of Theorem 1. We note that [23] is concerned with the time-optimal control problem. However, the relevant arguments from [23] carry over to the present setting with no significant changes. In fact, the Hamiltonians for Problem 1 and for the time-optimal problem are the same (modulo an additive constant), which makes their analysis quite similar. The reader is referred to [23] as well as $[2,9]$ for more details on the terminology and concepts involved. 
Let $L$ denote the Lie algebra generated by the vector fields $\boldsymbol{f}_{0}, \boldsymbol{g}_{1}, \ldots, \boldsymbol{g}_{m}$ (or, equivalently, the vector fields $\left.\boldsymbol{f}_{0}, \boldsymbol{f}_{1}, \ldots, \boldsymbol{f}_{m}\right)$. Under the assumption that these vector fields are analytic, $\mathbb{R}^{n}$ is partitioned into maximal integral manifolds of $L$, whose tangent spaces are given by $L$. Let us again consider Problem 1 for some initial state $\boldsymbol{p} \in \mathbb{R}^{n}$ and final time $t_{f}<T_{\max }(|\boldsymbol{p}|)$. Let $\tilde{\boldsymbol{u}}$ be an optimal control and $\tilde{\boldsymbol{x}}$ be the corresponding state trajectory. Then this trajectory is entirely contained in the maximal integral manifold $S$ of $L$ passing through $\boldsymbol{p}$. For each $\boldsymbol{x} \in S$, the tangent space to $S$ at $\boldsymbol{x}$ is $L(\boldsymbol{x})$. The Maximum Principle applied to the restriction of the system (8) to $S$ asserts the existence of a costate function $t \mapsto \tilde{\boldsymbol{\lambda}}(t)$ which satisfies the adjoint equation and is nontrivial on $L(\tilde{\boldsymbol{x}}(t))$ for all $t \in\left[0, t_{f}\right]$.

Next, let $L_{0}$ be the ideal in $L$ generated by $\boldsymbol{g}_{1}, \ldots, \boldsymbol{g}_{m}$ and let $S_{0}$ be the maximal integral manifold of $L_{0}$ passing through $\boldsymbol{p}$. As will become clear below, we cannot use Lemma 3 to prove that $\tilde{\boldsymbol{u}}$ is bang-bang unless $\tilde{\boldsymbol{\lambda}}(t)$ is nontrivial on $L_{0}(\tilde{\boldsymbol{x}}(t))$. The dimension of $L_{0}(\tilde{\boldsymbol{x}}(t))$ is constant and equal to either $\operatorname{dim} L(\tilde{\boldsymbol{x}}(t))$ or $\operatorname{dim} L(\tilde{\boldsymbol{x}}(t))-1$. We see that unless $\operatorname{dim} L_{0}(\tilde{\boldsymbol{x}}(t))=\operatorname{dim} L(\tilde{\boldsymbol{x}}(t))$, our optimal control $\tilde{\boldsymbol{u}}$ is not necessarily bang-bang. To handle this difficulty, we bring in an additional concept.

Following [23], we call a trajectory $\overline{\boldsymbol{x}}(\cdot ; \boldsymbol{p}, \overline{\boldsymbol{v}})$ of (8) strongly extremal if its control $\overline{\boldsymbol{v}}$ pointwise maximizes the Hamiltonian $H(\overline{\boldsymbol{x}}, \cdot, \overline{\boldsymbol{\lambda}})$ defined via (10) for some costate function $t \mapsto \overline{\boldsymbol{\lambda}}(t)$ which satisfies the adjoint equation and has a nontrivial restriction to the subspace $L_{0}(\overline{\boldsymbol{x}}(t))$ for all $t$ in its domain. Note that unlike for the costate $\tilde{\boldsymbol{\lambda}}$ appearing in the Maximum Principle of Section 2, no boundary condition is imposed on the costate $\overline{\boldsymbol{\lambda}}$ appearing in the definition of strong extremality. The notion of strong extremality does not directly correspond to optimality in the sense of Problem 1 (we will see later that it is related to a suitable auxiliary time-optimal control problem). However, the Hamiltonian maximization condition implies as before that the components of the control $\overline{\boldsymbol{v}}$ must almost everywhere satisfy

$$
\begin{array}{ll}
\bar{v}_{i}(t)=0 & \text { if } \psi_{i}(\overline{\boldsymbol{x}}(t), \overline{\boldsymbol{\lambda}}(t))<0 \text { or } \psi_{i}(\overline{\boldsymbol{x}}(t), \overline{\boldsymbol{\lambda}}(t))<\psi_{j}(\overline{\boldsymbol{x}}(t), \overline{\boldsymbol{\lambda}}(t)) \text { for some } j \neq i \\
\bar{v}_{i}(t)=1 & \text { if } \left.\psi_{i}(\overline{\boldsymbol{x}}(t), \overline{\boldsymbol{\lambda}}(t))\right)>0 \text { and } \psi_{i}(\overline{\boldsymbol{x}}(t), \overline{\boldsymbol{\lambda}}(t))>\psi_{j}(\overline{\boldsymbol{x}}(t), \overline{\boldsymbol{\lambda}}(t)) \text { for all } j \neq i
\end{array}
$$

with $\psi_{i}$ as defined in (12). In other words, since the boundary condition for $\tilde{\boldsymbol{\lambda}}$ was not used in deriving (13), controls corresponding to strongly extremal trajectories retain this important property of optimal controls.

The usefulness of strong extremality for us is that $\tilde{\boldsymbol{x}}$ can be replaced by a concatenation of a strong extremal and a trajectory corresponding to an arbitrary constant control, such that the final state at $t=t_{f}$ remains the same (hence the new control is also optimal). In fact, we have the following more general result, which is essentially a global version of [23, Lemma 2].

Lemma 4 Assume that the hypotheses of Theorem 1 hold. Consider an arbitrary trajectory $\boldsymbol{x}(t), t \in\left[0, t_{f}\right]$ of the system (8) with $\boldsymbol{x}(0)=\boldsymbol{p}$, and let $\boldsymbol{q}:=\boldsymbol{x}\left(t_{f}\right)$. Let $\boldsymbol{u}^{0}=\left(u_{1}^{0}, \ldots, u_{m}^{0}\right)^{T}$ be an arbitrary vertex of $\mathcal{U}$. Then there exists a time $\bar{t} \in\left[0, t_{f}\right]$ and an admissible control $\overline{\boldsymbol{v}}:[0, \bar{t}] \rightarrow \mathcal{U}$ with the following properties:

1. The trajectory $\boldsymbol{x}(t ; \boldsymbol{p}, \overline{\boldsymbol{v}}), t \in[0, \bar{t}]$ of $(8)$ is strongly extremal.

2. The solution of (8) with $\boldsymbol{x}(0)=\boldsymbol{p}$ corresponding to the control

$$
\overline{\boldsymbol{u}}(t)= \begin{cases}\overline{\boldsymbol{v}}(t) & \text { if } t \in[0, \bar{t}] \\ \boldsymbol{u}^{0} & \text { if } t \in\left(\bar{t}, t_{f}\right]\end{cases}
$$

satisfies $\boldsymbol{x}\left(t_{f} ; \boldsymbol{p}, \overline{\boldsymbol{u}}\right)=\boldsymbol{q}$.

Proof (sketch). Let $\boldsymbol{\phi}(\cdot, \boldsymbol{x})$ denote the integral curve of the vector field $\boldsymbol{f}_{0}+\sum_{k=1}^{m} \boldsymbol{g}_{k} u_{k}^{0} \in L$ passing through $\boldsymbol{x} \in \mathbb{R}^{n}$ at $t=0$. This is well defined for all $t$ and all $\boldsymbol{x}$ because it is the integral curve of one of the original complete vector fields $\boldsymbol{f}_{0}, \boldsymbol{f}_{1}, \ldots, \boldsymbol{f}_{m}$. Let $\boldsymbol{h}_{k}(t, \boldsymbol{x}):=\boldsymbol{\phi}(-t, \cdot)_{*} \boldsymbol{g}_{k}(\boldsymbol{\phi}(t, \boldsymbol{x})), k=1, \ldots, m$, where $\phi(t, \cdot)_{*}$ is the differential of the map $\phi(t, \cdot)$. Then $\boldsymbol{h}_{k}(t, \boldsymbol{x}) \in L_{0}(\boldsymbol{x})$ for all $k$ and all $t$. Let $\boldsymbol{\delta}_{\boldsymbol{v}}(t)$, 
$t \in\left[0, t_{f}\right]$ be a trajectory of (8) corresponding to some control $\boldsymbol{v}=\left(v_{1}, \ldots, v_{m}\right)^{T}$, with $\boldsymbol{\delta}_{\boldsymbol{v}}(0)=\boldsymbol{p}$. Let $\boldsymbol{\eta}_{\boldsymbol{v}}$ be the solution of the system

$$
\dot{\boldsymbol{x}}(t)=\sum_{k=1}^{m}\left(v_{k}(t)-u_{k}^{0}\right) \boldsymbol{h}_{k}(t, \boldsymbol{x}(t))
$$

with the same initial condition $\boldsymbol{\eta}_{\boldsymbol{v}}(0)=\boldsymbol{p}$. Then it can be directly verified that we have

$$
\boldsymbol{\delta}_{\boldsymbol{v}}(t)=\boldsymbol{\phi}\left(t, \boldsymbol{\eta}_{\boldsymbol{v}}(t)\right), \quad t \in\left[0, t_{f}\right] .
$$

(This is the so-called "variations formula", which is a nonlinear extension of the variation-of-constants formula for linear systems.)

The system (18) is a time-varying control system, with the same controls as (8). In particular, associated to our trajectory $\boldsymbol{x}(t), t \in\left[0, t_{f}\right]$ we have the trajectory $\boldsymbol{\eta}_{\boldsymbol{u}}(t), t \in\left[0, t_{f}\right]$ of $(18)$, where $\boldsymbol{u}$ is the control that generates $\boldsymbol{x}$ (so that $\boldsymbol{\delta}_{\boldsymbol{u}}=\boldsymbol{x}$ ). Let $\boldsymbol{q}^{\prime}:=\boldsymbol{\eta}_{\boldsymbol{u}}\left(t_{f}\right)$. Now we can choose, for the system (18), a time-optimal control $\overline{\boldsymbol{v}}$ which steers $\boldsymbol{p}$ to $\boldsymbol{q}^{\prime}$ in minimal time $\bar{t} \leq t_{f}$ (this control exists because our assumptions guarantee that $\boldsymbol{\eta}_{\boldsymbol{v}}$ is well defined on $\left[0, t_{f}\right]$ for all $\boldsymbol{v}$; cf. pages $633-634$ in [23]). By (19), this control $\overline{\boldsymbol{v}}$ then steers the state of the original system (8) from $\boldsymbol{p}$ to $\boldsymbol{\phi}\left(\bar{t}, \boldsymbol{q}^{\prime}\right)$. Repeating the arguments on page 634 in [23], we can show that the resulting trajectory of (8) is strongly extremal. (The basic idea is that by time-optimality of $\overline{\boldsymbol{v}}$ we have a costate for the system (18) which is nontrivial on $L_{0}\left(\boldsymbol{\eta}_{\overline{\boldsymbol{v}}}\right)$ and which can be "lifted" to produce a desired costate $\overline{\boldsymbol{\lambda}}$ for the original system.) Finally, the control (17) steers the state of (8) from $\boldsymbol{p}$ to $\boldsymbol{\phi}\left(t_{f}-\bar{t}, \boldsymbol{\phi}\left(\bar{t}, \boldsymbol{q}^{\prime}\right)\right)=\boldsymbol{\phi}\left(t_{f}, \boldsymbol{q}^{\prime}\right)=\boldsymbol{\delta}_{\boldsymbol{u}}\left(t_{f}\right)=\boldsymbol{q}$.

Remark 3 There are three minor differences between the above result and [23, Lemma 2]. First, Lemma 2 in [23] is stated for a time-optimal trajectory; however, the result is actually true for every trajectory (and in particular for an optimal trajectory $\tilde{\boldsymbol{x}}$ corresponding to Problem 1). Second, the treatment in [23] is limited to the single-input control system

$$
\dot{\boldsymbol{x}}=\boldsymbol{f}(\boldsymbol{x})+\boldsymbol{g}(\boldsymbol{x}) u, \quad|u| \leq 1
$$

but the above result remains valid for the multiple-input system (8). Finally, the construction given in [23] works on a small time interval, so that the overall trajectory is replaced by a concatenation of several pairs of strong extremals and constant-control trajectories, while the present completeness assumptions guarantee the existence of just one pair on the given time interval (see also the remark on page 635 in [23]).

Remark 4 The purpose of considering strongly extremal trajectories of the system (8) is to avoid singular trajectories and establish the existence of bang-bang optimal controls with a bound on the total number of switches. This task will be completed in Lemma 5 by using Lemmas 3 and 4 and the formula (16). Alternatively, it is possible to achieve the same goal by applying the Maximum Principle directly to the time-optimal control problem for the system (18) and obtaining a bound on the number of switches for the time-optimal control $\overline{\boldsymbol{v}}$. Then strong extremality of the "lifted" trajectory $\boldsymbol{x}(\cdot ; \boldsymbol{p}, \overline{\boldsymbol{v}})$ of the original control system would not be needed (and the proof could be made more self-contained). Yet another possibility, which would let us avoid any explicit use of the variations formula, is to construct an argument along the lines of the proof of Theorem 8.1.2 in [24].

Remark 5 It is clear that Lemma 4 remains valid if $t_{f}$ is replaced by an arbitrary time in $\left[0, t_{f}\right]$. In view of this and the fact that the system (8) is time-invariant, we immediately obtain the following generalization of Lemma 4 which will be needed in the sequel. Consider an arbitrary trajectory $\boldsymbol{x}(t), t \in\left[0, t_{f}\right]$ of $(8)$ with $\boldsymbol{x}(0)=\boldsymbol{p}$, and take two points $\boldsymbol{a}:=\boldsymbol{x}\left(t_{1}\right)$ and $\boldsymbol{b}:=\boldsymbol{x}\left(t_{2}\right), 0 \leq t_{1}<t_{2} \leq t_{f}$ on it. Let $\boldsymbol{u}^{0}$ be an arbitrary 
vertex of $\mathcal{U}$. Then there exists a time $\bar{t} \in\left[t_{1}, t_{2}\right]$ and a control $\overline{\boldsymbol{v}}:\left[t_{1}, \bar{t}\right] \rightarrow \mathcal{U}$ such that the concatenated control

$$
\overline{\boldsymbol{u}}(t)= \begin{cases}\overline{\boldsymbol{v}}(t) & \text { if } t \in\left[t_{1}, \bar{t}\right] \\ \boldsymbol{u}^{0} & \text { if } t \in\left(\bar{t}, t_{2}\right]\end{cases}
$$

transfers the state of (8) from $\boldsymbol{a}$ at time $t_{1}$ to $\boldsymbol{b}$ at time $t_{2}$ and the restriction of the resulting trajectory to $\left[t_{1}, \bar{t}\right]$ is strongly extremal.

We say (cf. [24]) that the control system (8) has the bang-bang property if, given an arbitrary trajectory $\boldsymbol{x}(t), t \in\left[0, t_{f}\right]$ of (8) with $\boldsymbol{x}(0)=\boldsymbol{p}$ and two points $\boldsymbol{a}:=\boldsymbol{x}\left(t_{1}\right)$ and $\boldsymbol{b}:=\boldsymbol{x}\left(t_{2}\right), 0 \leq t_{1}<t_{2} \leq t_{f}$ on it, there exists a bang-bang control that transfers the state from $\boldsymbol{a}$ at time $t_{1}$ to $\boldsymbol{b}$ at time $t_{2}$. By time-invariance of (8), this depends only on the difference $t_{2}-t_{1}$ and not on the individual times $t_{1}$ and $t_{2}$. If in addition there is an upper bound $N$, uniform over all trajectories and all $t_{2}-t_{1} \in\left[0, t_{f}\right]$, on the total number of switches of such bang-bang controls, then we say that (8) has the bang-bang property with $N$ switches.

In [23], Sussmann used an analog of Lemma 4 to derive a condition on the Lie brackets of $\boldsymbol{f}$ and $\boldsymbol{g}$ which guarantees the bang-bang property of (20), with bounds on the number of switches on finite time intervals. Bang-bang theorems for multiple-input nonlinear control systems, along similar lines but with additional hypotheses, are also available; see [3, 25]. However, the bang-bang property of (8) is not enough for proving that the switched system (1) is GUAS, because the number of switches may grow with the length of the time interval. To establish Theorem 1, we need to have a bound on the number of switches which is independent of time, i.e., we need to show that the control system (8) has the bang-bang property with $N$ switches for some $N$. The hypotheses of Theorem 1 are stronger than the conditions imposed in the above papers, and indeed allow us to reach such a conclusion. This is shown in the next lemma, which is of independent interest and will be our main tool in proving Theorem 1. Similarly to Lemma 4, this is a result about arbitrary trajectories, which includes the desired property of optimal trajectories as a special case.

Lemma 5 Under the hypotheses of Theorem 1, the system (8) has the bang-bang property with $N:=$ $(r+1)^{m}-1$ switches.

Proof $^{4}$. Consider two points $\boldsymbol{a}=\boldsymbol{x}\left(t_{1}\right)$ and $\boldsymbol{b}=\boldsymbol{x}\left(t_{2}\right)$ on an arbitrary trajectory $\boldsymbol{x}$ with $\boldsymbol{x}(0)=\boldsymbol{p}$. We want to show that there is a control that steers the state of (8) from $\boldsymbol{a}$ to $\boldsymbol{b}$ in time $t_{2}-t_{1}$, takes values in the vertices of $\mathcal{U}$, and has at most $(r+1)^{m}-1$ switches. This will imply the statement of the lemma. The number of vertices of $\mathcal{U}$ is $m+1$. Similarly to the proof of Theorem 8.1.2 in [24], we proceed by induction on $m$. If $m=0$, then $\mathcal{U}$ is a singleton and the statement is obvious. We now treat the case of an arbitrary $m>0$, supposing that the above property with $m-1$ instead of $m$ would hold for two arbitrary points on every trajectory of $(8)$ if $\mathcal{U}$ were a simplex with $m$ vertices. Apply Lemma 4 and Remark 5 to find another trajectory connecting $\boldsymbol{a}$ and $\boldsymbol{b}$, which is a concatenation of a strongly extremal trajectory $\overline{\boldsymbol{x}}(t), t \in\left[t_{1}, \bar{t}\right]$ generated by a control $\overline{\boldsymbol{v}}$ and a trajectory generated by a fixed vertex $\boldsymbol{u}^{0}$ of $\mathcal{U}$ for $t \in\left(\bar{t}, t_{2}\right]$. The costate function $\overline{\boldsymbol{\lambda}}$ associated with $\overline{\boldsymbol{x}}$ is nontrivial on $L_{0}(\overline{\boldsymbol{x}})$. Using (5) and (6) with $l=0$ together with (9), we have

$$
L_{0}=\operatorname{span}\left\{\left(\operatorname{ad} \boldsymbol{f}_{0}\right)^{s}\left(\boldsymbol{g}_{i}\right): i=1, \ldots, m, s=0, \ldots, r-1\right\} .
$$

Considering the functions $\psi_{i}, i=1, \ldots, m$ defined by (12) and using the formulas for their derivatives obtained in the proof of Lemma 3 , we see that the functions $\psi_{i}(\overline{\boldsymbol{x}}(\cdot), \overline{\boldsymbol{\lambda}}(\cdot))$ cannot all be identically zero. To simplify the notation, denote $\psi_{i}(\overline{\boldsymbol{x}}(t), \overline{\boldsymbol{\lambda}}(t))$ by $\bar{\varphi}_{i}(t)$.

\footnotetext{
${ }^{4}$ We thank an anonymous reviewer for identifying a gap in an earlier version of this proof.
} 
Suppose first that there exist some $i \neq j$ for which $\bar{\varphi}_{i}-\bar{\varphi}_{j}$ is not identically zero. By Lemma $3, \bar{\varphi}_{i}-\bar{\varphi}_{j}$ is a polynomial function of degree at most $r-1$. Hence, the time interval $\left[t_{1}, \bar{t}\right]$ is divided into at most $r$ subintervals, inside some of which we have $\bar{\varphi}_{i}(t)-\bar{\varphi}_{j}(t)<0$ and inside the others $\bar{\varphi}_{i}(t)-\bar{\varphi}_{j}(t)>0$. The formula (16) implies that the control $\overline{\boldsymbol{v}}$ satisfies $\bar{v}_{i}=0$ on subintervals of the former type and $\bar{v}_{j}=0$ on subintervals of the latter type (almost everywhere). Consider one such subinterval $\left[\tau_{1}, \tau_{2}\right] \subset\left[t_{1}, \bar{t}\right]$. Let $\overline{\boldsymbol{a}}:=\overline{\boldsymbol{x}}\left(\tau_{1}\right)$ and $\overline{\boldsymbol{b}}:=\overline{\boldsymbol{x}}\left(\tau_{2}\right)$. Assume, for example, that $\bar{\varphi}_{i}-\bar{\varphi}_{j}$ is negative on $\left(\tau_{1}, \tau_{2}\right)$. We have just shown that the restriction of $\overline{\boldsymbol{x}}$ to $\left[\tau_{1}, \tau_{2}\right]$ is a trajectory of (8) with controls taking values in the set $\mathcal{U}^{i}:=\left\{\boldsymbol{u} \in \mathcal{U}: u_{i}=0\right\}$. This set is a simplex with $m$ vertices (which are also vertices of $\mathcal{U}$ ). By the induction hypothesis, there is a control that steers the state of (8) from $\overline{\boldsymbol{a}}$ to $\overline{\boldsymbol{b}}$ in time $\tau_{2}-\tau_{1}$, takes values in the set of vertices of $\mathcal{U}^{i}$, and is a concatenation of at most $(r+1)^{m-1}$ constant controls. A similar conclusion holds for the restriction of $\overline{\boldsymbol{x}}$ to every other subinterval.

We also need to treat the case when each $\bar{\varphi}_{i}-\bar{\varphi}_{j}$ is identically zero, i.e., when all functions $\bar{\varphi}_{i}$ are identically equal to some constant $c$. We know that $c$ cannot be 0 . If $c<0$, then by (16) we have $\overline{\boldsymbol{v}} \equiv 0$ (almost everywhere). If $c>0$, then the fact that $\overline{\boldsymbol{v}}$ maximizes $H(\overline{\boldsymbol{x}}, \cdot, \overline{\boldsymbol{\lambda}})$ implies that $\bar{v}_{1}+\cdots+\bar{v}_{m} \equiv 1$ (almost everywhere), in other words, $\overline{\boldsymbol{v}}$ takes values in the set $\mathcal{U}^{0}:=\left\{\boldsymbol{u} \in \mathcal{U}: u_{1}+\cdots+u_{m}=1\right\}$. This set is again a simplex with $m$ vertices which are also vertices of $\mathcal{U}$. It does not contain the zero vertex, but we can bring the resulting control system back to the desired form by simply relabeling the vector fields $\boldsymbol{f}_{0}, \boldsymbol{f}_{1}, \ldots, \boldsymbol{f}_{m}$ so that $\boldsymbol{f}_{0}$ corresponds to one of the vertices of $\mathcal{U}^{0}$ (recall that the conditions (5) and (6) hold for all $l$, so $l=0$ does not have any special property). By the induction hypothesis, the state of (8) can be steered from $\boldsymbol{a}$ to $\overline{\boldsymbol{x}}(\bar{t})$ in time $\bar{t}-t_{1}$ by a concatenation of at most $(r+1)^{m-1}$ constant controls.

We have shown that the time interval $\left[t_{1}, \bar{t}\right]$ can be divided into at most $r$ subintervals, on each of which there exists a control which is a concatenation of at most $(r+1)^{m-1}$ constant controls, such that the overall control steers the state of (8) from $\boldsymbol{a}$ to $\overline{\boldsymbol{x}}(\bar{t})$. Applying the control $\boldsymbol{u}^{0}$ on the interval $\left(\bar{t}, t_{2}\right]$, we conclude that we can steer the state of the system from $\boldsymbol{a}$ to $\boldsymbol{b}$ in time $t_{2}-t_{1}$ by a bang-bang control which is a concatenation of at most $r(r+1)^{m-1}+1 \leq(r+1)^{m}$ constant controls, and the induction step is complete.

For $m>1$ the bound on the number of switches provided by Lemma 5 is conservative, as is clear from the proof. However, all we need is the fact that - in contrast with the result of [23] — this bound is independent of the final time $t_{f}$.

\section{Proof of Theorem 1}

We are now ready to prove the main result stated in the Introduction. Consider the control system (8). Each vertex of $\mathcal{U}$ corresponds to the closed-loop system $\dot{\boldsymbol{x}}=\boldsymbol{f}_{i}(\boldsymbol{x})$ for some index $i$ between 0 and $m$. This system is globally asymptotically stable, hence its solutions satisfy $|\boldsymbol{x}(t)| \leq \beta_{i}(|\boldsymbol{x}(0)|, t)$ for some class $\mathcal{K} \mathcal{L}$ function $\beta_{i}$. Let $\bar{\beta}:=\max \left\{\beta_{i}: i=0, \ldots, m\right\} \in \mathcal{K} \mathcal{L}$. Let $\alpha$ be the class $\mathcal{K}$ function defined by $\alpha(r):=\bar{\beta}(r, 0)$.

Consider Problem 1 with arbitrary $\boldsymbol{p}$ and $t_{f}<T_{\max }(|\boldsymbol{p}|)$. By Lemmas 2 and 5 , there exists a piecewise constant optimal control $\tilde{\boldsymbol{u}}:\left[0, t_{f}\right] \rightarrow \mathcal{U}$ that takes values in the vertices of $\mathcal{U}$ and has at most $N=$ $(r+1)^{m}-1$ switches. The interval $\left[0, t_{f}\right]$ is thus divided into at most $N+1$ subintervals, on each of which the optimal trajectory $\tilde{\boldsymbol{x}}$ satisfies one of the equations $\dot{\boldsymbol{x}}=\boldsymbol{f}_{i}(\boldsymbol{x}), i=0, \ldots, m$. The length of at least one of these subintervals is no smaller than $t_{f} /(N+1)$. Considering all possible locations of this subinterval relative to the others, it is straightforward to check that $\tilde{\boldsymbol{x}}$ satisfies

$$
\left|\tilde{\boldsymbol{x}}\left(t_{f}\right)\right| \leq \beta\left(|\boldsymbol{p}|, t_{f}\right)
$$


where

$$
\beta(r, t):=\max \left\{\alpha^{k}\left(\bar{\beta}\left(\alpha^{l}(r), t /(N+1)\right)\right): k, l \geq 0, k+l=N\right\}
$$

is a class $\mathcal{K} \mathcal{L}$ function. (Here $\alpha^{k}$ denotes the composition of $\alpha$ with itself $k$ times.)

All the desired conclusions follow from the formula (22). Since $\tilde{\boldsymbol{x}}$ is an optimal trajectory for Problem 1 which consists in maximizing $\left|\boldsymbol{x}\left(t_{f}\right)\right|^{2}$, it is clear that every other solution of (8) with $\boldsymbol{x}(0)=\boldsymbol{p}$ also satisfies $\left|\boldsymbol{x}\left(t_{f}\right)\right| \leq \beta\left(|\boldsymbol{p}|, t_{f}\right)$. Note that $N$, and consequently $\beta$, do not depend on $\boldsymbol{p}$ or $t_{f}$. In view of the bound $\beta\left(|\boldsymbol{p}|, t_{f}\right) \leq \beta(|\boldsymbol{p}|, 0)$ and the fact that $\boldsymbol{p}$ and $t_{f}<T_{\max }(|\boldsymbol{p}|)$ were arbitrary, we conclude that all solutions of (8) are bounded and so exist globally in time. Therefore, $t_{f}$ could be an arbitrary positive number, and all solutions of (8) satisfy the bound (4). We know from Lemma 1 that the same is automatically true for the solutions of the differential inclusion (3), which in turn include all solutions of the switched system (1). It remains to recall that (4) defines the stability properties that needed to be established.

Remark 6 It is clear from (21) that $L_{0}$ is a finite-dimensional Lie algebra, hence so is $L$ (because $\operatorname{dim} L \leq$ $\left.\operatorname{dim} L_{0}+1\right)$. Hector Sussmann has pointed out to us that in view of this fact, completeness of the differential inclusion (3) is guaranteed if the vector fields $\boldsymbol{f}_{0}, \boldsymbol{f}_{1}, \ldots, \boldsymbol{f}_{m}$ which generate $L$ are complete. This means that in fact there is no need to take $t_{f}$ to be small initially. (Note also that the special case of piecewise constant switching in (3) is covered by Palais' Theorem [18, Chapter IV, Theorem III] which guarantees completeness of all convex combinations of $\boldsymbol{f}_{0}, \boldsymbol{f}_{1}, \ldots, \boldsymbol{f}_{m}$.)

Remark 7 The analyticity of the vector fields $\boldsymbol{f}_{0}, \boldsymbol{f}_{1}, \ldots, \boldsymbol{f}_{m}$ was only needed to ensure that the Lie algebras $L$ and $L_{0}$ have the maximal integral manifold property (see the beginning of Section 3 ). This property also holds for vector fields that are only smooth, provided that $L(\boldsymbol{x})$ and $L_{0}(\boldsymbol{x})$ are assumed to be of constant rank on $\mathbb{R}^{n}$. Thus the analyticity hypothesis can be relaxed accordingly. See, e.g, [9, Chapter 2] for more details on this issue.

Remark 8 In the above proof we applied Lemma 5 to the optimal trajectory, but this lemma is valid for arbitrary trajectories. Thus optimality of $\tilde{\boldsymbol{x}}$ was not important in the proof and was used just for concreteness. In other words, we have established a general reachability result with a bound on the total number of switches, which includes the desired property of optimal trajectories as a special case and, combined with global asymptotic stability of the individual subsystems, directly implies Theorem 1. (However, the notion of strong extremality and the Maximum Principle did play a crucial role in the construction.)

\section{Conclusions}

We have formulated the first stability criterion for switched nonlinear systems (or, more generally, differential inclusions) that involves Lie brackets of the individual vector fields but does not require that these vector fields commute (Theorem 1). As a special case, we have shown that a switched system generated by two globally asymptotically stable nonlinear vector fields whose third-order Lie brackets vanish is globally uniformly asymptotically stable (Corollary 1). This represents a nonlinear extension of an earlier result from [7] and a promising step toward a solution of the open problem described in [11]. In contrast with the methods previously used to obtain results of this kind, our approach relied on reducing the stability analysis problem to the "worst-case" optimal control problem and then proving, via the Maximum Principle, the existence of an optimal control which is bang-bang with a bound on the total number of switches.

Acknowledgments. We are grateful to J. L. Mancilla-Aguilar and H. Sussmann for helpful discussions. 


\section{References}

[1] A. A. Agrachev and D. Liberzon. Lie-algebraic stability criteria for switched systems. SIAM J. Control Optim., 40:253-269, 2001.

[2] A. A. Agrachev and Yu. L. Sachkov. Control Theory from the Geometric Viewpoint. Springer, Berlin, 2004.

[3] A. A. Agrachev and S. A. Vakhrameev. Nonlinear control systems of constant rank and bang-bang conditions for extremal controls. Soviet Math. Dokl., 30:620-624, 1984.

[4] J.-P. Aubin and A. Cellina. Differential Inclusions: Set-Valued Maps and Viability Theory. Springer, Berlin, 1984.

[5] U. Boscain. Stability of planar switched systems: the linear single input case. SIAM J. Control Optim., 41:89-112, 2002.

[6] A. F. Filippov. Differential Equations with Discontinuous Righthand Sides. Kluwer, Dordrecht, 1988.

[7] L. Gurvits. Stability of discrete linear inclusion. Linear Algebra Appl., 231:47-85, 1995.

[8] D. Holcman and M. Margaliot. Stability analysis of switched homogeneous systems in the plane. SIAM J. Control Optim., 41(5):1609-1625, 2003.

[9] V. Jurdjevic. Geometric Control Theory. Cambridge University Press, 1996.

[10] D. Liberzon. Switching in Systems and Control. Birkhäuser, Boston, 2003.

[11] D. Liberzon. Lie algebras and stability of switched nonlinear systems. In V. Blondel and A. Megretski, editors, Unsolved Problems in Mathematical Systems and Control Theory, pages 203-207. Princeton University Press, 2004.

[12] D. Liberzon, J. P. Hespanha, and A. S. Morse. Stability of switched systems: a Lie-algebraic condition. Systems Control Lett., 37:117-122, 1999.

[13] Y. Lin, E. D. Sontag, and Y. Wang. A smooth converse Lyapunov theorem for robust stability. SIAM J. Control Optim., 34:124-160, 1996.

[14] J. L. Mancilla-Aguilar. A condition for the stability of switched nonlinear systems. IEEE Trans. Automat. Control, 45:2077-2079, 2000.

[15] M. Margaliot and R. Gitizadeh. The problem of absolute stability: a dynamic programming approach. Automatica, 40:1247-1252, 2004.

[16] M. Margaliot and G. Langholz. Necessary and sufficient conditions for absolute stability: the case of second-order systems. IEEE Trans. Circuits Syst.-I, 50:227-234, 2003.

[17] K. S. Narendra and J. Balakrishnan. A common Lyapunov function for stable LTI systems with commuting A-matrices. IEEE Trans. Automat. Control, 39:2469-2471, 1994.

[18] R. S. Palais. A global formulation of the Lie theory of transformation groups. Mem. Amer. Math. Soc., 22:1-123, 1957.

[19] E. S. Pyatnitskiy and L. B. Rapoport. Criteria of asymptotic stability of differential inclusions and periodic motions of time-varying nonlinear control systems. IEEE Trans. Circuits Syst.-I, 43:219-229, 1996.

[20] L. B. Rapoport. Asymptotic stability and periodic motions of selector-linear differential inclusions. In F. Garofalo and L. Glielmo, editors, Robust Control via Variable Structure and Lyapunov Techniques, pages 269-285. LNCIS 217, Springer, 1996.

[21] H. Shim, D. J. Noh, and J. H. Seo. Common Lyapunov function for exponentially stable nonlinear systems. J. Korean Institute of Electrical Engineers, 11:108-111, 2001. (Presented at the 4th SIAM Conf. on Control and its Applications, 1998).

[22] M. Sigalotti. Local regularity of optimal trajectories for control problems with general boundary conditions. $J$. Dynam. Control Systems, 11:91-123, 2005. 
[23] H. J. Sussmann. A bang-bang theorem with bounds on the number of switchings. SIAM J. Control Optim., 17:629-651, 1979.

[24] H. J. Sussmann. Lie brackets, real analyticity and geometric control. In R. W. Brockett, R. S. Millman, and H. J. Sussmann, editors, Differential Geometric Control Theory, pages 1-116. Birkhäuser, Boston, 1983.

[25] S. A. Vakhrameev. A bang-bang theorem with a finite number of switchings for nonlinear smooth control systems. J. Math. Sci., 85:2002-2016, 1997.

[26] R. Vinter. Optimal Control. Birkhäuser, Boston, 2000.

[27] L. Vu and D. Liberzon. Common Lyapunov functions for families of commuting nonlinear systems. Systems Control Lett., 54:405-416, 2005. 